\title{
The Effect of Frailty versus Initial Glasgow Coma Score in Predicting Outcomes Following Chronic Subdural Hemorrhage: A Preliminary Analysis
}

Matthew K. McIntyre ${ }^{1,2}$, Cameron Rawanduzy ${ }^{2}$, Adil Afridi ${ }^{2}$, Jesse A. Honig ${ }^{2}$, Mohamed Halabi ${ }^{2}$, Jake Hehir $^{2}$, Meic Schmidt ${ }^{3}$, Chad Cole ${ }^{4}$, Ivan Miller ${ }^{5}$, Chirag Gandhi ${ }^{4}$, Fawaz Al-Mufti ${ }^{4}$, Christian A. Bowers ${ }^{3}$

1. Department of Neurological Surgery, Oregon Health \& Science University, Portland, USA 2. Department of Neurosurgery, New York Medical College, Valhalla, USA 3. Department of Neurosurgery, University of New Mexico, Albuquerque, USA 4. Department of Neurosurgery, Westchester Medical Center, Valhalla, USA 5. Department of Emergency Medicine, Westchester Medical Center, Valhalla, USA

Corresponding author: Christian A. Bowers, christianbowers4@gmail.com

\section{Abstract \\ Background}

Initial Glasgow Coma Score (iGCS) is a well-known predictor of adverse outcomes following chronic subdural hemorrhage (cSDH). Frailty, i.e. a reduced physiologic reserve, is associated with poorer outcomes across the surgical literature, however, there is no consensus on the best measure of frailty. To date, no study has compared frailty's ability to predict cSDH outcomes versus iGCS. The goal of this study was to, therefore, examine the prognostic value of the 5 - (mFI-5) and 11-factor (mFI-11) modified frailty index, and Charlson Comorbidity Index (CCI) versus iGCS following cSDH.

\section{Methods}

Between January, 2016 and June, 2018, patients who presented to the emergency department with cSDH were retrospectively identified using the International Classification of Diseases (ICD) codes. mFI-5, mFI-11, and CCI scores were calculated using patient baseline characteristics. Primary endpoints were death and discharge home and subgroup analyses were performed among operative cSDH. Univariate and multivariate logistic regressions were used to determine predictors of primary endpoints.

\section{Results}

Of the 109 patients identified, the average age was $72.6 \pm 1.6$ years and the majority $(69 / 109,63.3 \%)$ were male. The average CCI, mFI- 5 , and $\mathrm{mFI}-11$ were $4.5 \pm 0.2,1.5 \pm 0.1$, and $2.2 \pm 0.1$, respectively. Fifty (45.9\%) patients required surgical intervention, 11 (10.1\%) died, and 48 (43.4\%) were discharged home. In the overall cohort, while the only multivariate predictor of mortality was iGCS (OR=0.58; $95 \% \mathrm{CI}: 0.44-0.77 ; \mathrm{p}=0.0001)$, the CCI (OR=0.73; 95\%CI:0.58-0.92; $\mathrm{p}=0.0082$ ) was a superior predictor of discharge home compared to iGCS

Received 08/04/2020

Review began 08/05/2020 Review ended 08/16/2020 Published 08/26/2020

\section{() Copyright 2020}

McIntyre et al. This is an open access article distributed under the terms of the Creative Commons Attribution License CC-BY 4.0., which permits unrestricted use, distribution, and reproduction in any medium, provided the original author and source are credited. ( $\mathrm{OR}=1.46$; 95\%CI:1.13-1.90; $\mathrm{p}=0.0041$ ). Conversely, among those who received an operative intervention, the CCI, but not iGCS, independently predicted both mortality (OR=4.24; 95\%CI:1.01-17.86; $\mathrm{p}=0.0491$ ) and discharge home (OR=0.55; 95\%CI:0.33-0.90; $\mathrm{p}=0.0170$ ). Neither $\mathrm{mFI}$ nor age predicted primary outcomes in multivariate analysis.

\section{Conclusion}

While frailty is associated with worse surgical outcomes, the clinical utility of the mFI-5, mFI-11, and CCI in cSDH is unclear. We show that the iGCS is an overall superior predictor of mortality following cSDH but is outperformed by the CCI after operative intervention. Similarly, the CCI is the superior predictor of discharge home in cSDH patients overall and following an operative intervention. These results indicate that while the iGCS best predicts mortality overall, the CCI may be considered when prognosticating postoperative course and hospital disposition.

Categories: Neurosurgery, Trauma

Keywords: modified frailty index, subdural hemorrhage, charlson comorbidity index, mortality, age, gcs

\section{Introduction}

Chronic subdural hemorrhage (CSDH) is an increasingly common pathology encountered in modern neurosurgical practice given its high frequency among the elderly [1]. The outcomes following cSDH are generally positive, but one large, recent cohort study found that age is correlated with poorer functional outcomes and lower rates of discharge home, and the majority of patients with cSDH are in their 80s [2]. While both advanced chronological age and low initial Glasgow Coma Score (iGCS) are established predictors of poorer outcomes following $\mathrm{CSDH},[2]$ there is a paucity of information regarding the effect of a patients' 
Frailty, i.e. a reduced physiologic reserve, is an emerging concept across the surgical literature that aims to elucidate the differential effects of age versus the cumulative effect of multiple comorbidities on surgical outcomes. While frailty, as most often measured by the modified frailty index (mFI-11), has been associated with poorer neurosurgical outcomes, its effect among those with cSDH is unclear [3-6]. To date, no study has compared the effect of frailty versus iGCS for predicting outcomes in both operative and non-operative cSDH. Complicating matters further, with over 215 different frailty indices in the literature [7], there remains no consensus regarding which frailty index best predicts outcomes; an effect that may be pathology and patient-population specific. Other common measures of frailty are the Charlson Comorbidity Index (CCI) and the new 5-factor modified frailty index (mFI-5). As such, the goal of this study is to perform a comparative analysis of multiple measures of frailty including the CCI, mFI-5, and mFI-11, versus iGCS for predicting mortality and discharge location among both operative and non-operative cSDH patients.

\section{Materials And Methods}

\section{Study design and setting}

This retrospective study was performed between January 2016 and June 2018 at a quaternary academic referral center (Westchester Medical Center) with high neurosurgical volume. Institutional Review Board Approval with a waiver of informed consent was obtained from New York Medical College \& Westchester Medical Center (IRB 12921). All data were retrospectively collected using the electronic medical record system by trained and monitored data abstractors.

\section{Subject selection}

cSDH patients were identified by reviewing the International Classification of Diseases (ICD) codes for subdural hemorrhage for all patients presenting to our emergency department in the study period. Inclusion criteria were patients 14 years or older who presented with a chronic subdural hemorrhage. Patients were excluded if they had a history of (non-subdural related) neurosurgical procedure or were found to not have a chronic SDH on in-house imaging. We defined cSDH as any subdural hemorrhage with a chronic component including mixed, acute on chronic, pure chronic, or subacute SDH.

\section{Measures and endpoints}

For each patient, demographics, smoking and alcohol abuse history, anti-coagulant or anti-platelet (AC/AP) drug use, GCS score, chief complaint, and cSDH characteristics (thickness, prior SDH), and presence of an isolated head injury were collected. The eleven-factor modified frailty index (mFI-11) was calculated by assigning one point for the presence of each of the following pre-hemorrhage characteristics for a maximum of 11 points: hypertension requiring medication, congestive heart failure, myocardial infarction, previous percutaneous coronary intervention or angina, transient ischemic attack or cerebrovascular accident without neurological deficit, cerebrovascular accident with neurological deficit, peripheral vascular disease or ischemic chest pain, chronic obstructive pulmonary disease or current pneumonia, diabetes mellitus, non-independent functional status, and impaired sensorium (Table 1). Non-independent functional status was defined as requiring assistance from another person for activities of daily living [8-11]. The five-factor modified frailty index (mFI-5) was defined similarly but with the presence of hypertension requiring medication, congestive heart failure, chronic obstructive pulmonary disease or current pneumonia, diabetes mellitus, or non-independent functional status [12,13]. The CCI was calculated as described extensively in prior literature (Table 2) [14,15]. 


\section{Cureus}

History of:

Hypertension on medications

Congestive Heart Failure

Diabetes mellitus

Chronic Obstructive Pulmonary Disease, Pneumonia

Non-independent functional status

History of transient ischemic attack or cerebrovascular accident without neurological deficit

Myocardial Infarction

Peripheral Vascular Disease or ischemic rest pain

Cerebral vascular accident with deficit

Previous coronary intervention or angina

Impaired Sensorium
$\mathrm{mFI}-5$

mFI-11

$77(70.6 \%)$

$14(12.8 \%)$

$24(22.0 \%)$

$9(8.3 \%)$

$35(32.1 \%)$

- $\quad 17(15.6 \%)$

- $\quad 9(8.3 \%)$

- $\quad 5(4.6 \%)$

- $\quad 9(8.3 \%)$

$-\quad 24(22.0 \%)$

- $\quad 21(19.3 \%)$

TABLE 1: 11- and 5-factor modified frailty index $(\mathrm{mFI})$ characteristics and prevalence in patients with chronic subdural hemorrhage (CSDH) 


\section{Cureus}

\begin{tabular}{|c|c|c|}
\hline Characteristic & Points & Prevalence \\
\hline \multicolumn{3}{|l|}{ Age (years) } \\
\hline$<50$ & 0 & $6(5.5 \%)$ \\
\hline $50-59$ & 1 & $18(16.5 \%)$ \\
\hline $60-69$ & 2 & $20(18.3 \%)$ \\
\hline 70-79 & 3 & $17(15.6 \%)$ \\
\hline$\geq 80$ & 4 & $48(44.0 \%)$ \\
\hline Myocardial Infarction & 1 & $9(8.3 \%)$ \\
\hline Congestive Heart Failure & 1 & $14(12.8 \%)$ \\
\hline Peripheral Vascular Disease & 1 & $5(4.6 \%)$ \\
\hline Cerebrovascular Accident or Transient Ischemic Attack & 1 & 17 (15.6\%) \\
\hline Dementia & 1 & 19 (17.4\%) \\
\hline Chronic Obstructive Pulmonary Disease & 1 & $9(8.3 \%)$ \\
\hline Connective Tissue Disease & 1 & $3(2.8 \%)$ \\
\hline Peptic Ulcer Disease & 1 & $5(4.6 \%)$ \\
\hline \multicolumn{3}{|l|}{ Liver Disease } \\
\hline Mild & 1 & $3(2.8 \%)$ \\
\hline Moderate to Severe & 3 & $5(4.6 \%)$ \\
\hline \multicolumn{3}{|l|}{ Diabetes Mellitus } \\
\hline Uncomplicated & 1 & $22(20.2 \%)$ \\
\hline End organ damage & 2 & $2(1.8 \%)$ \\
\hline Hemiplegia & 2 & $2(1.8 \%)$ \\
\hline Moderate to Severe Chronic Kidney Disease & 2 & 14 (12.8\%) \\
\hline \multicolumn{3}{|l|}{ Solid tumor } \\
\hline Localized & 2 & $13(11.9 \%)$ \\
\hline Metastatic & 6 & $1(0.9 \%)$ \\
\hline Leukemia & 2 & $3(2.8 \%)$ \\
\hline Lymphoma & 2 & $1(0.9 \%)$ \\
\hline AIDS & 6 & $0(0.0 \%)$ \\
\hline
\end{tabular}

TABLE 2: Charlson Comorbidity Index (CCI) characteristics and prevalence in patients with chronic subdural hemorrhage (cSDH)

Primary endpoints were discharge home and death. Secondary endpoints needed for surgical intervention (craniotomy, burr hole craniotomy, or subdural evacuating port system (SEPS) treatment), hospital length of stay (hLOS), ICU length of stay (ICU-LOS), deep vein thrombosis (DVT), pulmonary embolism (PE), pneumonia, tracheostomy, gastrostomy, and discharge GCS (excluding deaths).

\section{Statistical analysis}

Normal distributions were determined using an Anderson-Darling normality test. T-tests were used for normally distributed continuous samples and Mann-Whitney tests were used for non-normally distributed continuous samples. Continuous data were shown using mean \pm standard error of the mean (SEM). Fisher's exact tests were used for binary variables and odds ratios (OR) are shown with 95\% confidence intervals 
(95\% CI). Subgroup analyses were conducted among those who required an operation. Similar to prior studies, multivariate logistic regressions were performed via the enter method using known predictors of outcomes sex, prior subdural hemorrhage, craniotomy, AC/AP use, iGCS, and SDH thickness followed by forward conditional addition of either mFI-11, mFI-5, or CCI to the model. Age was not included due to collinearity with the CCI. No collinearity was detected in either model, as defined as a variance inflation factor of less than one or greater than 10. Statistical analysis was performed using Prism 8.3.0 (GraphPad Software Inc., La Jolla, CA) and SPSS version 25 (IBM Corp., Armonk, NY). Significance was defined as $\mathrm{p}<0.05$.

\section{Results}

\section{Demographics and baseline characteristics}

Of the 429 records identified, 109 met inclusion and exclusion criteria (Figure 1). The average age (range: 1498 years) of the cohort was $72.6 \pm 1.6$ years, the majority of patients were men (69/109, 63.3\%), and 25 (63.3\%) had a history of prior subdural hemorrhage (Table 3). As expected, the most common chief complaint was fall $(74 / 109,67.9 \%)$ and half of the patients had taken AC/AP medication prior to hemorrhage (55/109, $50.5 \%$ ). The average iGCS was $13.4 \pm 0.3$ (range: $3 \mathrm{~T}-15$ ) and the majority of patients had an isolated head injury (100/109, 91.7\%). Most hemorrhages were of mixed density (93/109, 85.3\%) and the average thickness was $16.0 \pm 0.7 \mathrm{~mm}$. For other baseline characteristics, see Table 3 .

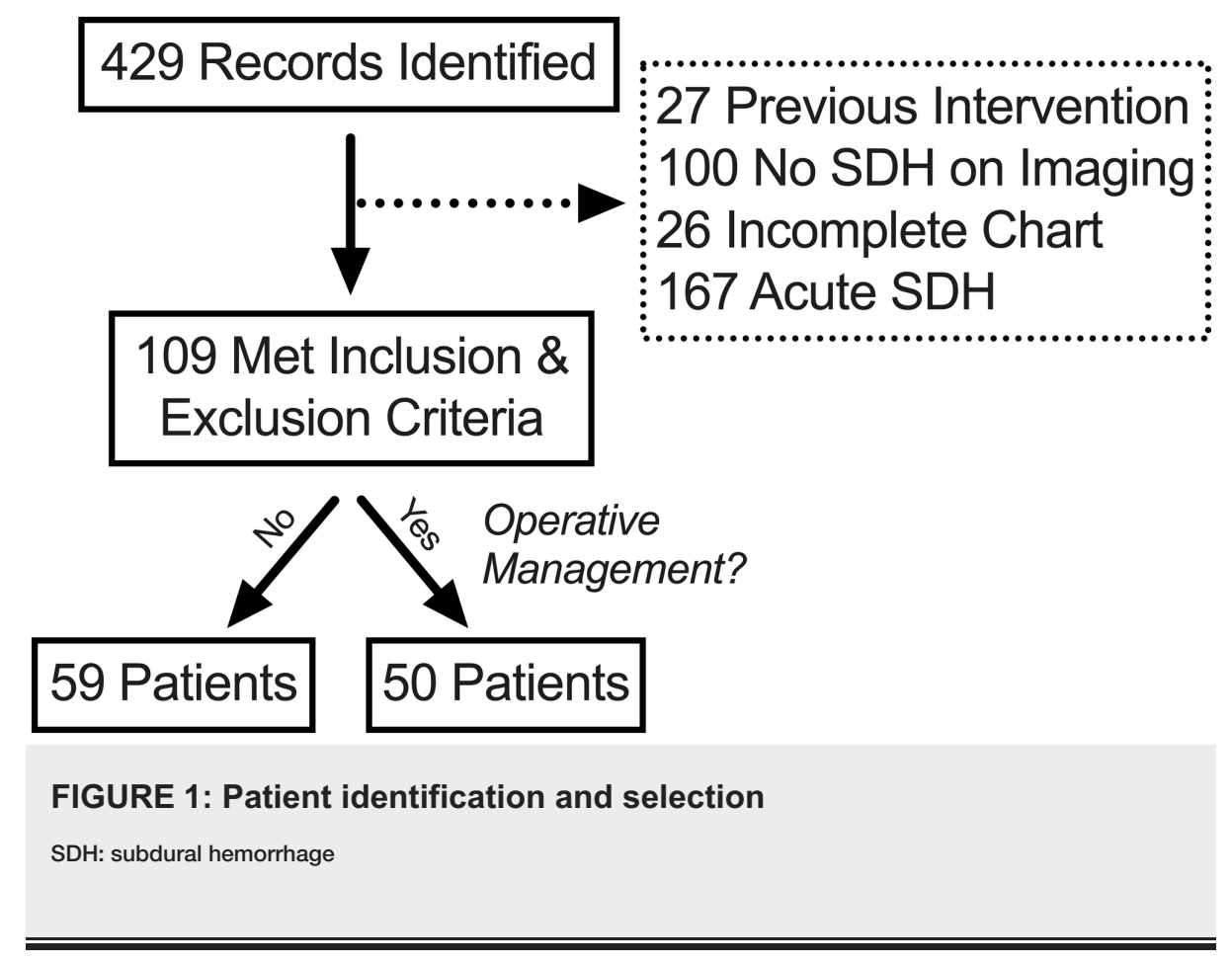




\section{Cureus}

\begin{tabular}{|c|c|c|c|c|}
\hline & Overall (n=109) & Non-operative $(n=59)$ & Operative $(n=50)$ & P-value \\
\hline Age (years)^ & $72.6 \pm 1.6$ & $76.5 \pm 2.1$ & $67.9 \pm 2.2$ & 0.0020 \\
\hline Men & 69 (63.3\%) & $39(57.4 \%)$ & $30(60.0 \%)$ & 0.8509 \\
\hline Prior subdural hemorrhage & $25(22.9 \%)$ & $14(23.7 \%)$ & $11(22.0 \%)$ & $>0.9999$ \\
\hline Smoking history & 39 (35.8\%) & $24(40.7 \%)$ & $15(30.0 \%)$ & 0.3167 \\
\hline Alcohol abuse & $18(16.5 \%)$ & $9(15.3 \%)$ & $9(18.0 \%)$ & 0.7978 \\
\hline AC/AP & 55 (50.5\%) & 27 (45.8\%) & $28(56.0 \%)$ & 0.3384 \\
\hline \multicolumn{5}{|l|}{ Chief Complaint } \\
\hline Fall & 74 (67.9\%) & $43(72.9 \%)$ & $31(62.0 \%)$ & 0.3034 \\
\hline Altered Mental Status & $12(11.0 \%)$ & 7 (11.9\%) & $5(10.0 \%)$ & $>0.9999$ \\
\hline MVC & $3(2.8 \%)$ & $0(0.0 \%)$ & $3(6.0 \%)$ & 0.0934 \\
\hline Assault & $2(1.8 \%)$ & $1(1.7 \%)$ & $1(2.0 \%)$ & $>0.9999$ \\
\hline Other/unknown & 20 (18.3\%) & $10(17.0 \%)$ & $10(20.0 \%)$ & 0.8050 \\
\hline iGCS $^{\wedge}$ & $13.4 \pm 0.3$ & $12.9 \pm 0.5$ & $13.9 \pm 0.3$ & 0.0423 \\
\hline Isolated head Injury & $100(91.7 \%)$ & $51(86.4 \%)$ & $49(98.0 \%)$ & 0.0370 \\
\hline cSDH thickness $(\mathrm{mm})^{\wedge}$ & $16.0 \pm 0.7$ & $13.6 \pm 0.9$ & $18.8 \pm 1.0$ & 0.0003 \\
\hline \multicolumn{5}{|l|}{ Type of cSDH } \\
\hline Mixed Density & $93(85.3 \%)$ & $49(83.1 \%)$ & $44(88.0 \%)$ & 0.5900 \\
\hline Subacute & $5(4.6 \%)$ & $4(6.8 \%)$ & $1(2.0 \%)$ & 0.3721 \\
\hline Pure Chronic & $11(10.1 \%)$ & $6(10.2 \%)$ & $5(10.0 \%)$ & $>0.9999$ \\
\hline $\mathrm{CCl}$ & $4.5 \pm 0.2$ & $5.1 \pm 0.3$ & $3.8 \pm 0.3$ & 0.0033 \\
\hline $\mathrm{mFI}-5^{\wedge}$ & $1.5 \pm 0.1$ & $1.5 \pm 0.1$ & $1.4 \pm 0.1$ & 0.2580 \\
\hline $\mathrm{mFl}-11^{\wedge}$ & $2.2 \pm 0.1$ & $2.5 \pm 0.2$ & $1.9 \pm 0.2$ & 0.0471 \\
\hline
\end{tabular}

\section{TABLE 3: Baseline characteristics and comparison between operative and non-operative patients}

^indicates non-normally distributed sample.

AC/AP: Anticoagulation/Antiplatelet medication, MVC: Motor Vehicle Collision, iGCS: initial Glasgow Coma Score, cSDH: chronic subdural

hemorrhage, $\mathrm{CCl}$ : Charlson Comorbidity Index, $\mathrm{mFI}$ : modified frailty index.

The average CCI was $4.5 \pm 0.2$ (range: 0 - 11 ) and 42 (38.5\%) had a $\mathrm{CCI}^{3} 6$. The most commonly identified CCI characteristic, beyond age, was a history of uncomplicated diabetes $(22 / 109,20.2 \%)$ followed by a history of dementia $(19 / 109,17.4 \%$ ) (Table 2). The average mFI-11 (range: 0-7) and mFI-5 (range: 0-4) was $2.2 \pm 0.1$ and $1.5 \pm 0.1$, respectively, with $73(67.0 \%)$ having a mFI- $11^{3} 2$ and 55 (50.5\%) having a mFI- $5^{3} 2$. The most commonly identified characteristic in both the $\mathrm{mFI}-5$ and $\mathrm{mFI}-11$ systems was hypertension on medications (77/109, 70.6\%) followed by non-independent functional status (35/109, 32.1\%) (Table 1).

\section{Clinical course and outcomes}

One hundred and five (96.3\%) patients were admitted and 50 (45.9\%) required a surgical evacuation of SDH. Five (4.6\%) patients developed a DVT, $1(0.9 \%)$ a PE, and $5(4.6 \%)$ pneumonia. Six $(5.5 \%)$ patients required a tracheostomy tube and 9 (8.3\%) a gastrostomy tube. The average ICU-LOS was $5.0 \pm 0.5$ days (range: $0-22$ ) while the hLOS was $9.0 \pm 0.9$ days (range: $1-62$ ). Ultimately, the average discharge GCS was $14.3 \pm 0.2$, a plurality of patients were discharged home (48/109, 44.0\%), and 11 (10.1\%) patients died (Table 4). 


\begin{tabular}{|c|c|c|c|c|}
\hline & Overall $(n=109)$ & Non-operative ( $n=59)$ & Operative (n=50) & P-value \\
\hline hLOS (days)^^ & $9.0 \pm 0.9$ & $8.3 \pm 1.4$ & $9.7 \pm 0.9$ & 0.0139 \\
\hline ICU LOS (days) & $5.0 \pm 0.5$ & $4.2 \pm 0.9$ & $5.7 \pm 0.7$ & 0.0011 \\
\hline Deep Vein Thrombosis & $5(4.6 \%)$ & $2(3.4 \%)$ & $3(6.0 \%)$ & 0.6591 \\
\hline Pulmonary Embolism & $1(0.9 \%)$ & $0(0.0 \%)$ & $1(2.0 \%)$ & 0.4588 \\
\hline Pneumonia & $5(4.6 \%)$ & $2(3.4 \%)$ & $3(6.0 \%)$ & 0.6591 \\
\hline Tracheostomy & $6(5.5 \%)$ & $3(5.1 \%)$ & $3(6.0 \%)$ & $>0.9999$ \\
\hline Gastrostomy & $9(8.3 \%)$ & $5(8.5 \%)$ & $4(8.0 \%)$ & $>0.9999$ \\
\hline Discharge GCS (without death) ^ & $14.3 \pm 0.2$ & $14.2 \pm 0.2$ & $14.5 \pm 0.2$ & 0.0793 \\
\hline \multicolumn{5}{|l|}{ Discharge location } \\
\hline Home & $48(44.0 \%)$ & $26(44.1 \%)$ & $22(44.0 \%)$ & $>0.9999$ \\
\hline Acute Rehab & $30(27.5 \%)$ & $9(15.3 \%)$ & $21(42.0 \%)$ & 0.0025 \\
\hline Subacute Rehab & $6(5.5 \%)$ & $4(6.8 \%)$ & $2(4.0 \%)$ & 0.6853 \\
\hline Nursing Home & $13(11.9 \%)$ & $12(20.3 \%)$ & $1(2.0 \%)$ & 0.0029 \\
\hline Death & $11(10.1 \%)$ & $7(11.9 \%)$ & $4(8.0 \%)$ & 0.543 \\
\hline
\end{tabular}

\section{TABLE 4: Patient Outcomes and comparison between operative and non-operative patients}

^indicates non-normally distributed sample.

hLOS: hospital length of stay, ICU-LOS: Intensive care unit length of stay.

\section{Clinical course and outcomes: the effect of operative management}

Patients who received operative evacuation of SDH were significantly younger $(67.9 \pm 2.2$ versus $76.5 \pm 2.1$; $\mathrm{p}=0.0020)$, had a higher iGCS ( $13.9 \pm 0.3$ versus $12.9 \pm 0.5$; $\mathrm{p}=0.0423)$, larger SDH thickness $(18.8 \pm 1.0$ versus $13.6 \pm 0.9 ; \mathrm{p}=0.0003$ ), and were more likely to have an isolated head injury (OR=7.7; 95\%CI: 1.1-86.9; $\mathrm{p}=0.0370$ ), indicating careful selection of surgical candidates. Likewise, those that received an operation had decreased CCI ( $3.8 \pm 0.3$ versus $5.1 \pm 0.3 ; \mathrm{p}=0.0033$ ) and $\mathrm{mFI}-11(1.9 \pm 0.2$ versus $2.5 \pm 0.2 ; \mathrm{p}=0.0370$ ) but not $\mathrm{mFI}-5(\mathrm{p}=0.2580)$ scores compared to those who did not have an operation. There were no significant baseline differences in history of SDH, smoking or alcohol abuse history, AC/AP use, or type of SDH between those who did or did not receive an intervention (Table 3).

As expected, those that received an operation had longer hospital ( $9.7 \pm 0.9$ versus $8.3 \pm 1.4$ days; $p=0.0139$ ) and ICU ( $5.7 \pm 0.7$ versus $4.2 \pm 0.9$ days; $p=0.0011$ ) length of stay but no differences in complications ( $p>0.05$ ) (Table 4). Interestingly, there were no differences in rates of discharge home or death between those who did or did not receive surgical drainage. However, patients in the operative group were more likely to be discharged to acute rehabilitation ( $\mathrm{OR}=4.0 ; 95 \% \mathrm{CI}: 1.7-9.2 ; \mathrm{p}=0.0025)$, less likely to be discharged to a nursing home ( $\mathrm{OR}=0.08 ; 95 \% \mathrm{CI}$ : 0.007-0.48; $\mathrm{p}=0.0029$ ), and trended to have a higher discharge $\mathrm{GCS}$ scores (14.5 \pm 0.2 versus $14.2 \pm 0.2 ; \mathrm{p}=0.0793$ ). Of the patients who received operative evacuation of SDH, 33 (66\%) had a craniotomy while 17 (34\%) had either a burr hole craniotomy or a subdural evacuation port system device placement. There were no significant differences in any primary or secondary endpoints between the two strategies of operative management (Table 4).

\section{Clinical course and outcomes: the effect of patient frailty}

As expected, patients in the $\mathrm{CCI} \geq 6, \mathrm{mFI}-11 \geq 2$, and $\mathrm{mFI}-5 \geq 2$ groups were each older than their non-frail counterparts (Table 5). Those in the CCI $\geq 6(\mathrm{p}=0.0080)$ and $\mathrm{mFI}-11 \geq 2(\mathrm{p}=0.0093)$ groups also presented with significantly lower iGCS scores. Those in the $\mathrm{mFI}-5 \geq 2$ group also trended to have decreased iGCS scores compared to those in the $\mathrm{mFI}-5 \leq 1$ group. As noted above, those that received an operation had significantly lower CCI and mFI-11 scores compared to those that did not receive an intervention (Table 4). Despite this, there were no significant differences in SDH thickness, hospital or ICU LOS, complications, tracheostomy, or gastrostomy tube need. Patients in the mFI- $11 \geq 2(\mathrm{p}=0.0108)$ and $\mathrm{mFI}-5 \geq 2(0.0186)$ groups, but not $\mathrm{CCI} \geq 6$, were discharged with significantly lower GCS scores. There were also significant reductions in rates of discharge home and corresponding increases in discharge to nursing home or hospice in each frailty group. Finally, while there were no significant differences in mortality, there were more deaths in each of the frail 


\section{Cureus}

groups.

\begin{tabular}{|c|c|c|c|c|c|c|c|c|c|c|}
\hline & & \multicolumn{3}{|l|}{ CCl } & \multicolumn{3}{|l|}{ mFI-11 } & \multicolumn{3}{|l|}{ mFI-5 } \\
\hline & & $\begin{array}{l}\mathrm{CCl} \leq 5 \\
(\mathrm{n}=67)\end{array}$ & $\begin{array}{l}\mathrm{CCl} \geq 6 \\
(\mathrm{n}=42)\end{array}$ & P-value & $\begin{array}{l}\mathrm{mFI} \leq 1 \\
(\mathrm{n}=36)\end{array}$ & $\begin{array}{l}\mathrm{mFI} \geq 2 \\
(\mathrm{n}=73)\end{array}$ & P-value & $\begin{array}{l}\mathrm{mFI}-\mathbf{5} \leq \mathbf{1} \\
(\mathrm{n}=54)\end{array}$ & $\begin{array}{l}\mathrm{mFI}-5 \geq 2 \\
(\mathrm{n}=55)\end{array}$ & $\mathrm{p}$-value \\
\hline \multicolumn{2}{|c|}{ Age (years) ^ } & $66.8 \pm 2.1$ & $81.8 \pm 1.6$ & $<0.0001$ & $66.1 \pm 3.3$ & $75.7 \pm 1.6$ & 0.0035 & $69.1 \pm 2.4$ & $75.9 \pm 1.9$ & 0.0349 \\
\hline \multicolumn{2}{|l|}{ iGCS^ } & $13.6 \pm 0.4$ & $13.0 \pm 0.4$ & 0.0080 & $14.2 \pm 0.4$ & $13.0 \pm 0.4$ & 0.0093 & $13.7 \pm 0.4$ & $13.1 \pm 0.4$ & 0.0850 \\
\hline \multicolumn{2}{|c|}{ SDH thickness } & $15.6 \pm 0.9$ & $16.5 \pm 1.2$ & 0.5550 & $16.4 \pm 1.3$ & $15.8 \pm 0.9$ & 0.6953 & $16.4 \pm 1.0$ & $15.5 \pm 1.0$ & 0.5363 \\
\hline \multicolumn{2}{|c|}{ hLOS (days)^ } & $9.7 \pm 1.2$ & $7.8 \pm 1.0$ & 0.2720 & $8.8 \pm 1.3$ & $9.0 \pm 1.1$ & 0.7781 & $7.9 \pm 1.0$ & $9.9 \pm 1.4$ & 0.4039 \\
\hline \multicolumn{2}{|c|}{ ICU LOS (days) } & $5.0 \pm 0.7$ & $4.9 \pm 0.9$ & 0.3948 & $4.8 \pm 0.8$ & $5.0 \pm 0.7$ & 0.4745 & $4.7 \pm 0.7$ & $5.2 \pm 0.9$ & 0.6792 \\
\hline \multicolumn{2}{|c|}{ Deep Vein Thrombosis } & $4(6.0 \%)$ & $1(2.4 \%)$ & 0.6470 & $1(2.8 \%)$ & $4(5.5 \%)$ & $>0.9999$ & $1(1.9 \%)$ & $4(7.3 \%)$ & 0.3634 \\
\hline \multicolumn{2}{|c|}{ Pulmonary Embolism } & $1(1.5 \%)$ & $0(0.0 \%)$ & $>0.9999$ & $0(0.0 \%)$ & $1(1.4 \%)$ & $>0.9999$ & $0(0.0 \%)$ & $1(1.8 \%)$ & $>0.9999$ \\
\hline \multicolumn{2}{|l|}{ Pneumonia } & $4(6.0 \%)$ & $1(2.4 \%)$ & 0.6470 & $1(2.8 \%)$ & $4(5.5 \%)$ & $>0.9999$ & $1(1.9 \%)$ & $4(7.3 \%)$ & 0.3634 \\
\hline \multicolumn{2}{|c|}{ Tracheostomy } & $5(7.5 \%)$ & $1(2.4 \%)$ & 0.4025 & $1(2.8 \%)$ & $5(6.8 \%)$ & 0.6616 & $2(3.7 \%)$ & $4(7.3 \%)$ & 0.6787 \\
\hline \multicolumn{2}{|c|}{ Gastrostomy } & $6(9.0 \%)$ & $3(7.1 \%)$ & $>0.9999$ & $3(8.3 \%)$ & $6(8.2 \%)$ & $>0.9999$ & $6(11.1 \%)$ & $3(5.5 \%)$ & 0.3203 \\
\hline \multicolumn{2}{|c|}{ Discharge GCS (without death)^ } & $14.3 \pm 0.2$ & $14.5 \pm 0.2$ & 0.5264 & $14.7 \pm 0.2$ & $14.1 \pm 0.2$ & 0.0108 & $14.7 \pm 0.1$ & $14.0 \pm 0.3$ & 0.0186 \\
\hline \multirow{4}{*}{$\begin{array}{l}\text { Discharge } \\
\text { Location }\end{array}$} & Home & $35(51.5 \%)$ & $13(31.0 \%)$ & 0.0477 & $\begin{array}{l}22 \\
(61.1 \%)\end{array}$ & $\begin{array}{l}26 \\
(35.6 \%)\end{array}$ & 0.0144 & 31 (57.4\%) & $17(30.9 \%)$ & 0.0070 \\
\hline & Acute Rehab & $22(32.8 \%)$ & $8(19.1 \%)$ & 0.1295 & $9(25.0 \%)$ & $\begin{array}{l}21 \\
(28.8 \%)\end{array}$ & 0.8204 & $13(24.1 \%)$ & $17(30.9 \%)$ & 0.5211 \\
\hline & Subacute Rehab & $2(3.0 \%)$ & $4(9.5 \%)$ & 0.2022 & $1(2.8 \%)$ & $5(7.9 \%)$ & 0.4121 & $3(5.6 \%)$ & $3(5.5 \%)$ & $>0.9999$ \\
\hline & $\begin{array}{l}\text { Nursing Home/ } \\
\text { hospice }\end{array}$ & $3(4.5 \%)$ & $10(23.8 \%)$ & 0.0045 & $1(2.8 \%)$ & $\begin{array}{l}12 \\
(16.4 \%)\end{array}$ & 0.0566 & $2(3.7 \%)$ & $11(20.0 \%)$ & 0.0153 \\
\hline \multicolumn{2}{|l|}{ Death } & $5(7.4 \%)$ & 6 (14.3\%) & 0.3281 & $2(5.6 \%)$ & $9(12.3 \%)$ & 0.3325 & $4(7.4 \%)$ & 7 (12.7\%) & 0.5269 \\
\hline
\end{tabular}

\section{TABLE 5: The effect of frailty on primary and secondary outcomes}

CCI: Charlson Comorbidity Index, mFI: modified frailty index, iGCS: initial Glasgow Coma Score, hLOS: hospital length of stay, ICU-LOS: Intensive Care Unit length of stay.

\section{Multivariate logistic regressions}

To examine the influence of each frailty measure as predictors of mortality and discharge home, stepwise multivariate logistic regressions were performed while including variables known to be associated with poorer outcomes following cSDH. Age was not included for this analysis due to co-linearity with frailty measures. In multivariate analysis, the single most significant predictor of mortality was the iGCS $(\mathrm{OR}=0.58$; 95\%CI: 0.44-0.77; $\mathrm{p}<0.0001$ ) and was not independently predicted by any frailty measure (Table 6 ). Discharge home, however, was independently predicted by both iGCS (OR=1.46; 95\%CI: 1.13-1.90; $\mathrm{p}=0.0041$ ) and $\mathrm{CCI}(\mathrm{OR}=0.73$; 95\%CI: 0.58-0.92; $\mathrm{p}=0.0082)$ score but not either $\mathrm{mFI}$. 


\section{Cureus}

\begin{tabular}{|c|c|c|c|}
\hline & Characteristic & Multivariate OR $(95 \% \mathrm{Cl})$ & P-value \\
\hline \multirow{9}{*}{ Mortality } & Sex & $2.03(0.26-15.60)$ & 0.4961 \\
\hline & Prior SDH & 0 (0-infinity) & 0.9980 \\
\hline & Craniotomy for SDH & $1.46(0.17-12.62)$ & 0.7310 \\
\hline & AC/AP & $1.31(0.19-8.89)$ & 0.7815 \\
\hline & iGCS & $0.58(0.44-0.77)$ & 0.0001 \\
\hline & SDH Thickness & $1.07(0.92-1.24)$ & 0.3605 \\
\hline & $\mathrm{mFI}-11$ & Not Included & 0.6560 \\
\hline & $\mathrm{mFI}-5$ & Not Included & 0.6387 \\
\hline & $\mathrm{CCl}$ & Not Included & 0.3296 \\
\hline \multirow{9}{*}{ Discharge Home } & Sex & $0.65(0.26-1.67)$ & 0.3716 \\
\hline & Prior SDH & $0.50(0.16-1.52)$ & 0.2218 \\
\hline & Craniotomy for SDH & $0.46(0.15-1.38)$ & 0.1647 \\
\hline & AC/AP & $1.55(0.58-4.15)$ & 0.3808 \\
\hline & iGCS & $1.46(1.13-1.90)$ & 0.0041 \\
\hline & SDH Thickness & $1.00(0.93-1.06)$ & 0.8932 \\
\hline & $\mathrm{mFl}-11$ & Not Included & 0.6812 \\
\hline & $\mathrm{mFI}-5$ & Not Included & 0.1833 \\
\hline & $\mathrm{CCl}$ & $0.73(0.58-0.92)$ & 0.0082 \\
\hline
\end{tabular}

TABLE 6: Multivariate logistic regressions for primary endpoints among all patients in the cohort

CCI: Charlson Comorbidity Index, mFI: modified frailty index, iGCS: initial Glasgow Coma Score, AC/AP: anticoagulation/antiplatelet medication.

\section{Subgroup analysis of operative patients}

Stepwise multivariate logistic regressions, in the same manner as above, were performed among those who received an operative intervention. Among this group, the only independent predictor of mortality was the CCI (OR=4.24; 95\%CI: 1.01-17.86; $\mathrm{p}=0.0491$ ) (Table 7). Similarly, the only independent predictor of discharge home among this group was also the CCI $(\mathrm{OR}=0.55 ; 95 \% \mathrm{CI}: 0.33-0.90 ; \mathrm{p}=0.0170)$. Unlike the cohort overall, the iGCS was not independently associated with either endpoint among those that received an operative intervention. 


\section{Cureus}

\begin{tabular}{|c|c|c|c|}
\hline & Characteristic & Multivariate OR $(95 \% \mathrm{Cl})$ & P-value \\
\hline \multirow{9}{*}{ Mortality } & Sex & $0.93(0.05-17.53)$ & 0.9615 \\
\hline & Prior SDH & 0 (0-infinity) & 0.9980 \\
\hline & Craniotomy for SDH & $0.34(0.01-16.75)$ & 0.5840 \\
\hline & AC/AP & infinity (0-infinity) & 0.9973 \\
\hline & iGCS & $1.17(0.67-2.02)$ & 0.5831 \\
\hline & SDH Thickness & $0.69(0.41-1.16)$ & 0.1606 \\
\hline & $\mathrm{mFI}-11$ & Not Included & 0.3296 \\
\hline & $\mathrm{mFI}-5$ & Not Included & 0.4914 \\
\hline & $\mathrm{CCl}$ & $4.24(1.01-17.86)$ & 0.0491 \\
\hline \multirow{9}{*}{ Discharge Home } & Sex & $2.08(0.45-9.69)$ & 0.3499 \\
\hline & Prior SDH & $0.20(0.03-1.57)$ & 0.1246 \\
\hline & Craniotomy for SDH & $0.58(0.12-2.81)$ & 0.4943 \\
\hline & AC/AP & $0.90(0.17-4.90)$ & 0.9039 \\
\hline & iGCS & $1.02(0.68-1.54)$ & 0.9128 \\
\hline & SDH Thickness & $1.05(0.94-1.17)$ & 0.3841 \\
\hline & $\mathrm{mFl}-11$ & Not Included & 0.7254 \\
\hline & $\mathrm{mFI}-5$ & Not Included & 0.5783 \\
\hline & $\mathrm{CCl}$ & $0.55(0.33-0.90)$ & 0.0170 \\
\hline
\end{tabular}

\section{TABLE 7: Multivariate logistic regressions for primary endpoints among operative cSDH patients}

CCI: Charlson Comorbidity Index, mFI: modified frailty index, iGCS: initial Glasgow Coma Score, AC/AP: anticoagulation/antiplatelet medication; cSDH: chronic subdural hemorrhage.

\section{Discussion}

Frailty predicts outcomes in a variety of surgical and non-surgical patients. Despite cSDH being a common neurosurgical condition associated with cerebral atrophy and advanced age, the utility of frailty has not been explored in this population. In this study, we show that frail patients, regardless of scoring system, are less likely to be discharged home compared to their non-frail counterparts. Along with iGCS, the CCI was independently associated with discharge home in multivariate analysis in the cohort overall and was the only independent predictor of this endpoint in patients who received an operation. Similarly, while mortality overall was best predicted by iGCS, the CCI was the only independent predictor of death in subgroup analysis of operative cases. These results indicate that the CCI may have clinical utility in predicting functional outcome in all cSDH patients and mortality among those who receive an operation.

It is well known that patient comorbidities contribute to poorer prognosis following a neurologic insult. Frailty is an emerging concept that aims to quantify the cumulative effect of these comorbidities in order to more accurately prognosticate outcomes. In neurosurgery specifically, frailty has been associated with poorer outcomes following subarachnoid hemorrhage [5,11], spine surgery [16], intracranial hemorrhage [17], and intra-cranial tumor surgery [18]. Despite this, there are over 215 established frailty indices [7] and no consensus on the optimal scoring system or appropriate cutoffs [19]; something that may be disease and patient-population specific. For example, in our own work we have shown in the same time period and setting as the study herein, the superiority of the $\mathrm{mFI}-5$ and $\mathrm{mFI}-11$ over the $\mathrm{CCI}$ in predicting outcomes following angiogram-negative subarachnoid hemorrhage (under review, BJN). This is in contrast to the study herein showing the opposite finding for $\mathrm{cSDH}$. This indicates that despite frailty being associated with poorer outcomes across all of neurosurgery [20], individual scoring systems must be validated for each disease type. 
hemorrhage size, MLS, age, overall clinical history and exam, and the iGCS. In particular, the iGCS following cSDH is thought to be the factor with the highest prognostic utility $[21,22]$. In line with this, patients in our study who had an operation were younger, were more likely to have an isolated head injury, a better iGCS, larger cSDH thickness, and lower CCI and mFI-11 scores (Table 3). Likewise, while patients who had an evacuation had longer lengths of stay, they were more likely to be discharged to acute rehabilitation, equally as likely to be discharged home, less likely to be discharged to a nursing home, and trended to have lower mortality compared to their non-operative counterparts. Moreover, among the subgroup of operative patients, the CCI was the only independent predictor of both mortality and discharge location. Together, this reflects careful patient selection for those who are likely to do well after surgery, but given the retrospective nature of this study, we cannot comment on the clinical utility of frailty in patient selection for surgery. Prospective studies with larger samples of those who received a craniotomy or burr hole only may provide more conclusive evidence that the CCI, in addition to better-documented criteria, may be considered for operative candidate selection.

While the mFI-11 is the most commonly used frailty measure in the literature, to date, no study has examined the mFI-11 or mFI-5 among patients with cSDH; an effect that may reflect a publication bias. Furthermore, while there have been previous studies investigating the relationship between the CCI and cSDH prognosis, there are conflicting results with some finding that the CCI is independently associated with cSDH recurrence [23], increased complications [24,25] and long-term mortality [26], while others have shown no such effects [2,27]. Differences in these studies may be attributed to inclusion criteria, with some groups, including our own, examining all cSDH patients, while others only examined certain types of operative patients. For example, Shimizu et al. retrospectively examined 211 patients $\geqslant 65$ years of age who underwent burr hole craniotomy to determine predictors of three month modified Rankin scores, non-home discharge, and unfavorable prognosis. This group showed, in multivariate analysis, that age, nutritional status, and recurrence better predicted endpoints than the clinical frailty score, an established functional measure of frailty, and the CCI. Of note, this group did not include iGCS in multivariate regressions and retrospectively calculated functional frailty scores based on documentation thus limiting the relative clinical utility of their findings [28]. In a similar limitation, we did not include age in multivariate analyses as this variable was included in the CCI score and to do so would incur co-linearity between these variables. Along with this, our goal was to compare frailty with the iGCS and we showed that the iGCS was the most potent predictor of both mortality and discharge home in cSDH overall but was inferior to the CCI among operative patients.

\section{Limitations}

This study was chiefly limited by its retrospective design as outlined elsewhere [29] and by a limited sample size. Larger samples may have elucidated more significant effects of frailty on secondary endpoints and perhaps on mortality. In the latter endpoint, there was a trend toward increased mortality with all examined frailty indices. Second, we included all patients who presented with SDH that had a chronic component including mixed, acute-on-chronic, and pure chronic SDH. Larger prospectively obtained cohorts would allow for subgroup analysis of each cSDH type and for stronger comparisons of craniotomy versus burr hole craniotomy.

\section{Conclusions}

While frailty is associated with worse surgical outcomes, the clinical utility of the mFI-5, mFI-11, and CCI versus the iGCS following cSDH is unclear. We show that the iGCS is an overall superior predictor of mortality following cSDH but is outperformed by the CCI after operative intervention. Similarly, the CCI is the superior predictor of discharge home in CSDH patients overall and following an operative intervention. These results indicate that while the iGCS best predicts mortality overall, the CCI may be considered when prognosticating post-operative course and hospital disposition.

\section{Additional Information \\ Disclosures}

Human subjects: Consent was obtained by all participants in this study. New York Medical College issued approval 12921. IRB approval for this retrospective study was obtained from New York Medical College with a waiver of informed consent. . Animal subjects: All authors have confirmed that this study did not involve animal subjects or tissue. Conflicts of interest: In compliance with the ICMJE uniform disclosure form, all authors declare the following: Payment/services info: All authors have declared that no financial support was received from any organization for the submitted work. Financial relationships: All authors have declared that they have no financial relationships at present or within the previous three years with any organizations that might have an interest in the submitted work. Other relationships: This work was supported, in part, by a grant from New York Medical College for medical student research.

\section{Acknowledgements}

We would like to thank Christine Loscri, Monique Carrero-Tangle, and Lizbel Aquino for their support of neurosurgical research. 


\section{References}

1. Rauhala M, Luoto TM, Huhtala H, Iverson GL, Niskakangas T, Öhman J, Helén P: The incidence of chronic subdural hematomas from 1990 to 2015 in a defined Finnish population. J Neurosurg. 2019, 132:1147-1157. 10.3171/2018.12.jns 183035

2. Raghavan A, Smith G, Onyewadume L, et al.: Morbidity and mortality after burrhole craniostomy versus craniotomy for chronic subdural hematoma evacuation: a single-center experience. World Neurosurg. 2020, 134:e196-e203. 10.1016/j.wneu.2019.10.023

3. Seib CD, Rochefort H, Chomsky-Higgins K, Gosnell JE, Suh I, Shen WT, Duh QY: Association of patient frailty with increased morbidity after common ambulatory general surgery operations. JAMA Surg. 2018, 153:160-168. 10.1001/jamasurg.2017.4007

4. Youngerman BE, Neugut AI, Yang J, Hershman DL, Wright JD, Bruce JN: The modified frailty index and 30day adverse events in oncologic neurosurgery. J Neurooncol. 2018, 136:197-206. 10.1007/s11060-017-26440

5. McIntyre M, Gandhi C, Dragonette J, et al.: Increasing frailty predicts worse outcomes and increased complications after angiogram-negative subarachnoid hemorrhages. World Neurosurg. 2020, 134:e181e188. 10.1016/j.wneu.2019.10.003

6. Velanovich V, Antoine H, Swartz A, Peters D, Rubinfeld I: Accumulating deficits model of frailty and postoperative mortality and morbidity: Its application to a national database. J Surg Res. 2013, 183:104-110. 10.1016/j.jss.2013.01.021

7. Costenoble A, Knoop V, Vermeiren S, et al.: A comprehensive overview of activities of daily living in existing frailty instruments: a systematic literature search. Gerontologist. 2019, gnz147. 10.1093/geront/gnz147

8. Saxton A, Velanovich V: Preoperative frailty and quality of life as predictors of postoperative complications . Ann Surg. 2011, 253:1223-1229. 10.1097/SLA.0b013e318214bce7

9. Tsiouris A, Hammoud ZT, Velanovich V, Hodari A, Borgi J, Rubinfeld I: A modified frailty index to assess morbidity and mortality after lobectomy. J Surg Res. 2013, 183:40-46. 10.1016/j.jss.2012.11.059

10. Shin JI, Kothari P, Phan K, Kim JS, Leven D, Lee NJ, Cho SK: Frailty index as a predictor of adverse postoperative outcomes in patients undergoing cervical spinal fusion. Spine. 2017, 42:304-310. 10.1097/BRS.0000000000001755

11. McIntyre MK, Gandhi C, Long A, et al.: Age predicts outcomes better than frailty following aneurysmal subarachnoid hemorrhage: a retrospective cohort analysis. Clin Neurol Neurosurg. 2019, 187:105558. 10.1016/j.clineuro.2019.105558

12. Subramaniam S, Aalberg JJ, Soriano RP, Divino CM: New 5-factor modified frailty index using American College of Surgeons NSQIP data. J Am Coll Surg. 2018, 226:173-181.e8. 10.1016/j.jamcollsurg.2017.11.005

13. Yagi M, Michikawa T, Hosogane N, et al.: The 5-Item modified frailty index is predictive of severe adverse events in patients undergoing surgery for adult spinal deformity. Spine. 2019, 44:E1083-E1091. 10.1097/BRS.0000000000003063

14. Meagher AD, Lin A, Mandell SP, Bulger E, Newgard C: A comparison of scoring systems for predicting shortand long-term survival after trauma in older adults. Acad Emerg Med. 2019, 26:621-630. 10.1111/acem.13727

15. Charlson M, Pompei P, Ales K, MacKenzie C: A new method of classifying prognostic comorbidity in longitudinal studies: development and validation. J Chronic Dis. 1987, 40:373-383. 10.1016/00219681(87)90171-8

16. Ali R, Schwalb JM, Nerenz DR, Antoine HJ, Rubinfeld I: Use of the modified frailty index to predict 30-day morbidity and mortality from spine surgery. J Neurosurg Spine. 2016, 25:537-541. 10.3171/2015.10.SPINE14582

17. Imaoka Y, Kawano T, Hashiguchi A, et al.: Modified frailty index predicts postoperative outcomes of spontaneous intracerebral hemorrhage. Clin Neurol Neurosurg. 2018, 175:137-143. 10.1016/j.clineuro.2018.11.004

18. Isobe N, Ikawa F, Tominaga A, et al.: Factors related to frailty associated with clinical deterioration after meningioma surgery in the elderly. World Neurosurg. 2018, 119:e167-e173. 10.1016/j.wneu.2018.07.080

19. Pazniokas J, Gandhi C, Theriault B, et al.: The immense heterogeneity of frailty in neurosurgery: a comprehensive literature review [Epub ahead of print]. Neurosurg Rev. 2020, 10.1007/s10143-020-01241-2

20. Tomlinson SB, Piper K, Kimmell KT, Vates GE: Preoperative frailty score for 30-day morbidity and mortality after cranial neurosurgery. World Neurosurg. 2017, 107:959-965. 10.1016/j.wneu.2017.07.081

21. Alford EN, Rotman LE, Erwood MS, et al.: Development of the Subdural Hematoma in the Elderly (SHE) score to predict mortality. J Neurosurg. 2019, 132:1616-1622. 10.3171/2019.1.jns182895

22. Kuhn EN, Erwood MS, Oster RA, Davis MC, Zeiger HE, Pittman BC, Fisher III WS: Outcomes of subdural hematoma in the elderly with a history of minor or no previous trauma. World Neurosurg. 2018, 119:e374e382. 10.1016/j.wneu.2018.07.168

23. Martinez-Perez R, Tsimpas A, Rayo N, Cepeda S, Lagares A: Role of the patient comorbidity in the recurrence of chronic subdural hematomas [Epub ahead of print]. Neurosurg Rev. 2020, 10.1007/s10143020-01274-7

24. Lee L, Ker J, Ng H, Munusamy T, King NKK, Kumar D, Ng WH: Outcomes of chronic subdural hematoma drainage in nonagenarians and centenarians: a multicenter study. J Neurosurg. 2016, 124:546-551. 10.3171/2014.12.JNS142053

25. Bartek J, Sjåvik K, Kristiansson H, Ståhl F, Fornebo I, Förander P, Jakola AS: Predictors of recurrence and complications after chronic subdural hematoma surgery: a population-based study. World Neurosurg. 2017, 106:609-614. 10.1016/j.wneu.2017.07.044

26. De Bonis P, Olei S, Mongardi L, et al.: Chronic subdural hematoma in patients aged 80 years and older: a two-centre study. Clin Neurol Neurosurg. 2018, 170:88-92. 10.1016/j.clineuro.2018.05.002

27. Franko LR, Sheehan KM, Roark CD, Joseph JR, Burke JF, Rajajee V, Williamson CA: A propensity score analysis of the impact of surgical intervention on unexpected 30-day readmission following admission for 


\section{Cureus}

subdural hematoma. J Neurosurg. 2018, 129:1008-1016. 10.3171/2017.6.JNS17188

28. Shimizu K, Sadatomo T, Hara T, Onishi S, Yuki K, Kurisu K: Importance of frailty evaluation in the prediction of the prognosis of patients with chronic subdural hematoma. Geriatr Gerontol Int. 2018, 18:1173-1176. 10.1111/ggi.13436

29. Kaji AH, Schriger D, Green S: Looking through the retrospectoscope: reducing bias in emergency medicine chart review studies. Ann Emerg Med. 2014, 64:292-298. 10.1016/j.annemergmed.2014.03.025 